\title{
Photochemical Charge Separation in Poly(3-hexylthiophene) (P3HT) Films Observed with Surface Photovoltage Spectroscopy
}

\author{
Frank E. Osterloh, ${ }^{* \dagger}{ }^{\dagger}$ Michael A. Holmes, ${ }^{\dagger}$ Lilian Chang, ${ }^{\ddagger}$ Adam J. Moulé, ${ }^{\dagger}$ and Jing Zhao ${ }^{\dagger}$ \\ ${ }^{\dagger}$ Department of Chemistry, University of California, Davis, California 95616, United States \\ ${ }^{\ddagger}$ Department of Chemical Engineering and Materials Science, University of California, Davis, California 95616, United States
}

ABSTRACT: Surface photovoltage spectroscopy (SPS) was used to probe photon induced charge separation in thin films of regioregular and regiorandom poly(3-hexylthiophene) (P3HT) as a function of excitation energy. Both positive and negative photovoltage signals were observed under sub-band-gap $(<2.0 \mathrm{eV})$ and super-band-gap $(>2.0 \mathrm{eV})$ excitation of the polymer. The dependence of the spectra on substrate work function, thermal annealing, film thickness, and illumination intensity was investigated, allowing the identification of interface, charge transfer (CT), and band-gap states in the amorphous and crystalline regions of the polymer films. The ability to probe these states in polymer films will aid the development and optimization of organic electronic devices such as photovoltaics (OPVs), light-emitting diodes (OLEDs), and field effect transistors (OFETs). The direction
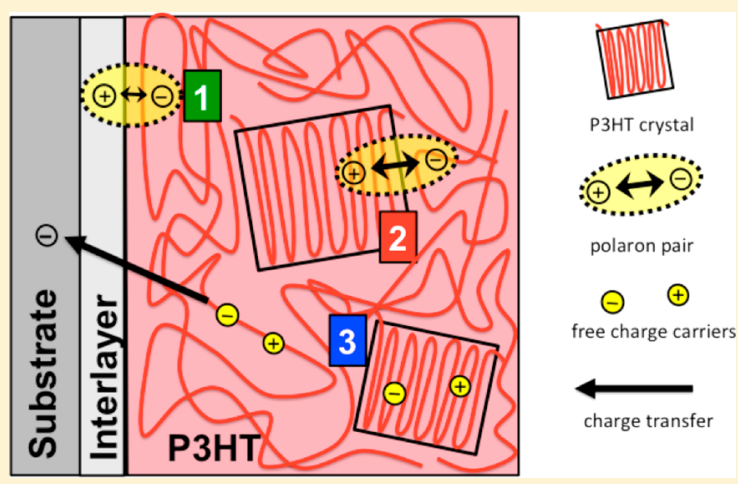
and size of the observed photovoltage features can be explained using the depleted semiconductor model.

\section{INTRODUCTION}

Organic photovoltaic (OPV) cells, light-emitting diodes (OLEDs), and field effect transistors (OFETs) rely on organic semiconductor polymers for creating, separating, and converting electrical charge. ${ }^{1-10}$ The mechanism of photochemical charge generation and transport in such materials has been of considerable interest. Recent work suggests that the so-called charge transfer (CT) state between the donor and the acceptor controls the open circuit voltage and photocurrent of OPV devices. ${ }^{11-20}$ Evidence supports that these CT states also occur within films of pure organic semiconductors ${ }^{21-23}$ as well as at any interfaces these polymers make with other materials. ${ }^{24,25}$

Interface and CT states have a very low absorption cross section, making them difficult or impossible to observe in UVvis-NIR absorption spectra of such polymers. ${ }^{26}$ But they have been detected through other methods including photoluminescence and photothermal deflection spectroscopy, ${ }^{27}$ Fourier transform photocurrent spectroscopy, ${ }^{15,26}$ electroluminescence, ${ }^{28}$ two-pulse femtosecond, ${ }^{16}$ and charge modulation spectroscopy. ${ }^{29}$

Here we demonstrate that the CT state and other important, but previously not detectable, interfacial states in organic polymers can be observed using surface photovoltage spectroscopy (SPS). SPS is a well-known tool for the observation of photoinduced charge separation within semiconductor materials. ${ }^{30-36}$ The method measures electrical potential, not current, making it sensitive to very small changes in the distribution of charge carriers. Also, as a contact-less technique, SPV does not require fully assembled devices, but rather films on a conductive substrate are sufficient. To date, there are only a few documented cases of the use of SPS on organic semi- conductors. ${ }^{37-39}$ For organic semiconductors the related Kelvin probe or electrostatic force microscopy techniques are generally preferred, ${ }^{40-44}$ even though they generally do not provide information on the spectral dependence of the transitions. ${ }^{41}$

Figure 1A shows the SPS measurement configuration. Thin film samples on conductive substrates are transferred into a vacuum chamber $\left(10^{-7} \mathrm{bar}\right)$, and the contact potential difference is measured with a vibrating Kelvin probe made of a semitransparent gold grid. In the dark, this electrical potential is mostly determined by the difference of the electrochemical potentials between the sample and the gold Kelvin probe $(\simeq$ $E_{\mathrm{F}}[1]-E_{\mathrm{F}}[2]$ ) (Figure 1B). ${ }^{30,35}$ In a semiconductor sample, illumination changes the local vacuum level of the sample due to the production and separation of charge carriers. This results in a photovoltage (plotted as CPD in Figures 2, 4, 5, 6, and 7A) whose spectral dependence can aid the understanding of photoactive states in polymer films and probe changes in interface dipoles that are difficult to access by other means. Detail of these processes (Figure 3C) will be discussed below.

Photovoltage measurements were conducted on thin films (Figure 1C) of poly(3-hexylthiophene) (P3HT), an important donor material for bulk-heterojunction (BHJ) $\mathrm{OPVs}^{43,45-47}$ and hole conductor for OFETs. ${ }^{48}$ Silver, gold, and ITO substrates with variable work function $(\Phi)$ and interlayer coatings were employed to probe the effect of interfacial dipoles and charge equilibria on photochemical charge separation in the P3HT films. Additionally, the effect of the P3HT linkage

Received: September 16, 2013

Revised: December 2, 2013

Published: December 2, 2013 


\section{A}

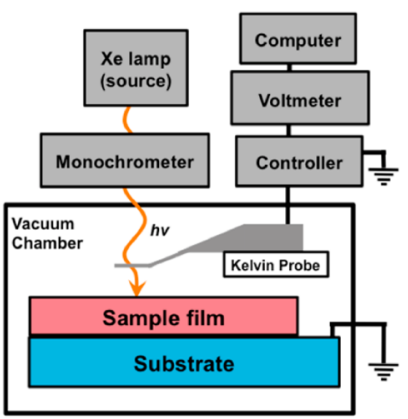

C



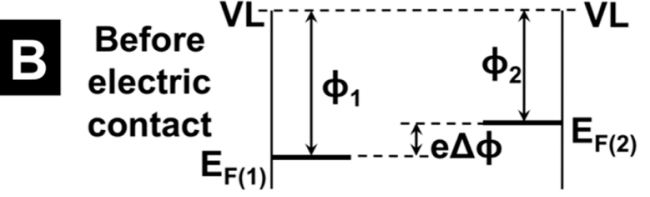

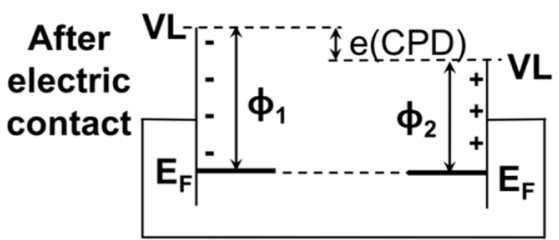

D
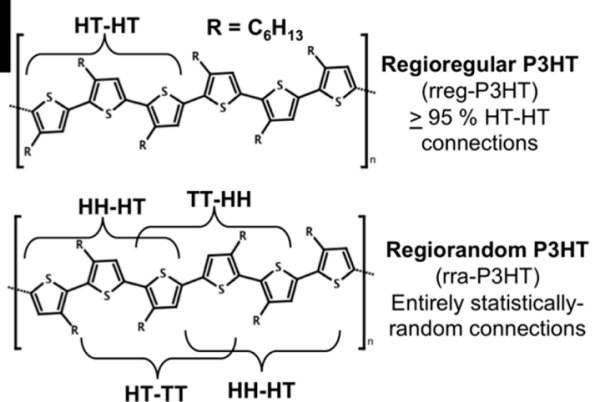

Figure 1. (A) Diagram of SPS measurement configuration. (B) Energy diagram for Kelvin probe (2) and a metallic sample (1) before and after electric contact with respective work functions $\phi_{i}$. The electrochemical equilibrium between 1 and 2 shifts the local vacuum levels and gives rise to the observed contact potential difference CPD. Adapted from Kronik. ${ }^{30,35}$ The energy scheme for P3HT films is shown in Figure 3C. (C) General makeup of sample films for SPS. Interlayers of PEDOT:PSS or amorphous $\mathrm{TiO}_{x}$ were present only when specifically noted in text. (D) Structure formulas of rr- and rra-P3HT. Monomer linkages occur through either the 2-position of the ring, the "head" $(\mathrm{H})$, or the 5-position, the "tail" $(\mathrm{T})$.
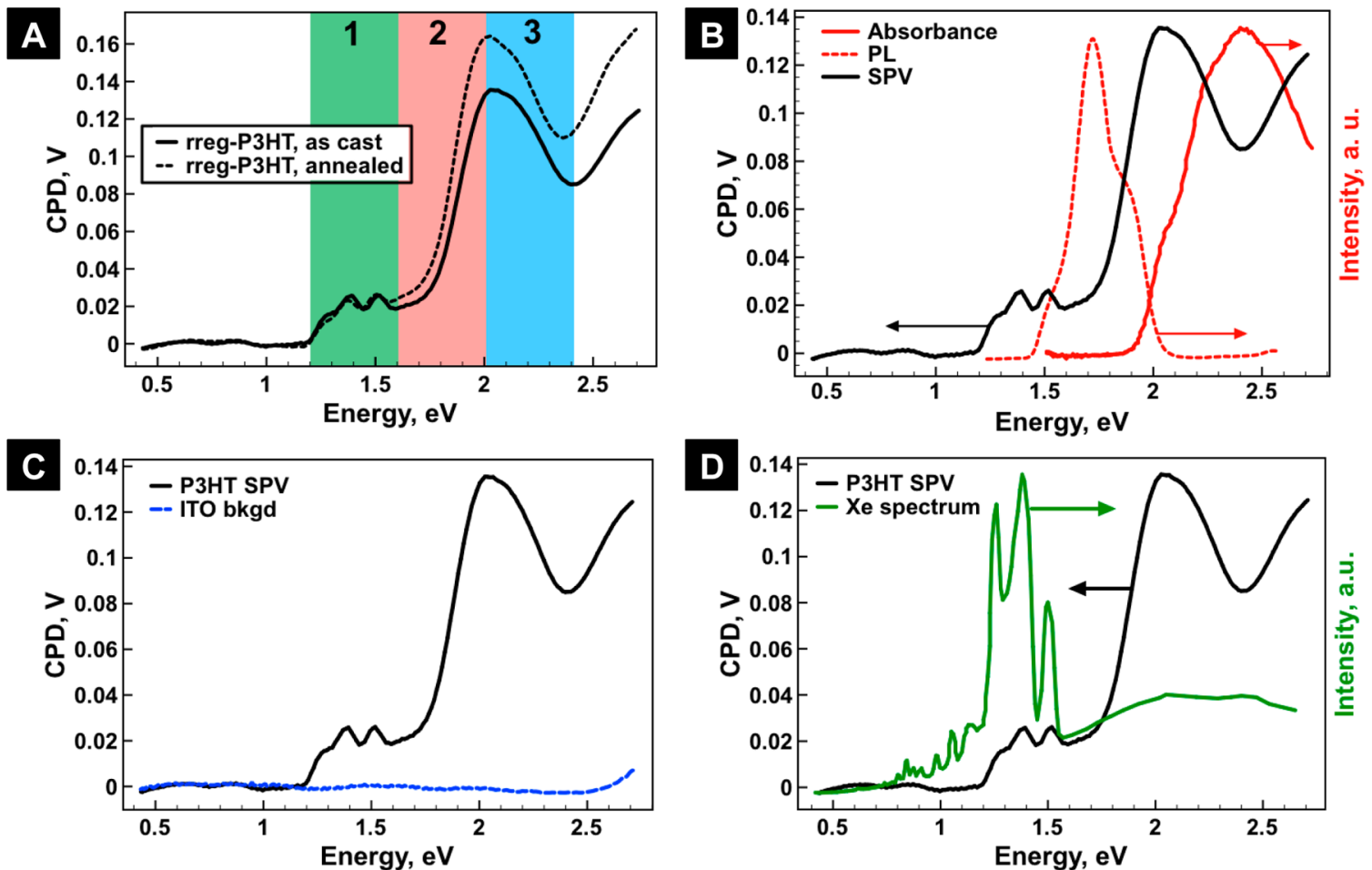

Figure 2. (A) Photovoltage spectrum (not normalized for light intensity) of a $120 \mathrm{~nm}$ thick, as-cast and annealed, rr-P3HT film on ITO with feature ranges color-coded and numbered. Feature 1 corresponds to the rise of the $\mathrm{CPD}$ value from 0 to $0.02 \mathrm{~V}$, feature 2 to the rise from 0.02 to $0.16 \mathrm{~V}$, and feature 3 to the decrease from 0.16 to $0.08 \mathrm{~V}$. (B) Photovoltage spectrum along with normalized UV-vis absorption and photoluminescence spectra of the annealed P3HT film in (A). (C) Photovoltage spectrum of rr-P3HT on ITO and of ITO-only background. (D) Xenon lamp emission and photovoltage spectra of rr-P3HT.

configuration was evaluated in the measurements. As Figure 1D shows, two forms of $\mathrm{P} 3 \mathrm{HT}$, regioregular (rr) and regiorandom (rra), can be distinguished depending on the connectivity between thiophene monomers (through the head at the 2- 
position or through the tail at the 5-position) (Figure 1D). RrP3HT with its many HT-HT linkages has a lower interchain distance $(0.38 \mathrm{~nm})^{29}$ and higher crystallinity than rra-P3HT, allowing strong interchain interactions to occur which can enhance interchain charge transport. Lastly, the SPV measurements probe the effect of thermal processing on the SPV spectra of P3HT. Heating is known to promote the formation of crystalline domains in P3HT films and to modify charge transport in the polymer. ${ }^{49-51}$

\section{RESULTS AND DISCUSSION}

Figure 2A shows SPV spectra for a $120 \mathrm{~nm}$ thick film of rrP3HT on ITO, before and after annealing at $120^{\circ} \mathrm{C}$. Spectra were corrected for background effects (drift, desorption of gas and solvent molecules) by subtracting a background scan in the dark. The spectra were recorded under steady-state conditions; i.e., neither changing the scan time length nor reversing the scan direction had an effect on the observed signals. Four main features can be distinguished, of which three (henceforth labeled 1,2 , and 3 ) originate from the polymer.

Feature 1 is a weak positive $\triangle \mathrm{CPD}$ signal $(0-26 \mathrm{mV})$ that begins at $1.0-1.2 \mathrm{eV}$, depending on substrate, and reaches to $1.7 \mathrm{eV}$. This signal is not present in the optical spectra of the polymer films. The fine structure of the signal is not due to the polymer but can be attributed to the spectral emission pattern of the xenon arc lamp (Figure 2D). Feature 2 is a positive CPD change in the spectrum $(26-136 \mathrm{mV})$ and occurs in the $1.7-$ $2.0 \mathrm{eV}$ energy range. This feature coincides with the energy interval of the photoluminescence emission of rr-P3HT films (Figure 2B), which has been previously ascribed to the polaron, or charge transfer state, in rr-P3HT. ${ }^{49,52,53}$ Feature 3 is found at photon energies of $2.0-2.4 \mathrm{eV}$ as a negative CPD change of intermediate size $(85-136 \mathrm{mV})$. It overlaps with the optical absorption of P3HT (Figure 2B) and can be assigned to the direct excitation of the HOMO-LUMO transition of the P3HT polymer chain. Feature 4 is observed at illumination energies $>2.4 \mathrm{eV}$; it was also observed, although weaker on a bare ITO substrate (Figure 2C), indicating that it does not originate from the polymer but is an artifact of the instrument.

As will be shown in the following, features 1-3 vary considerably with substrate, film thickness, polymer type and on the basis of these observations can be assigned to specific excitation processes in the film, as shown in Figure 3A. A justification for the assignment is given in the following.

The low excitation energy of feature 1 rules out band gap excitation of the polymer or excitation of the CT state. The signal is instead assigned to the excitation of interfacial charge transfer states (ICT) at the polymer-substrate interface. Accordingly, the size and magnitude of the photovoltage vary with the work function of the substrate material. For example, the signal is small $(30 \mathrm{mV})$ for ITO and occurs at $1.2 \mathrm{eV}$, whereas the voltage is large $(80 \mathrm{mV})$ on gold and red-shifted to $1.1 \mathrm{eV}$ (Figure 4A). On silver, the photovoltage begins at 1.0 $\mathrm{eV}$, but again is small $(20 \mathrm{mV}$, Figure $4 \mathrm{~B})$. When a $40 \mathrm{~nm}$ thick PEDOT:PSS layer is inserted between the P3HT film and the ITO substrate, the photovoltage doubles $(44 \mathrm{mV})$, in comparison to the bare ITO sample (Figure 4C). Conversely, when a $20 \mathrm{~nm}$ annealed layer of amorphous $\mathrm{TiO}_{x}$ is deposited onto ITO before the addition of P3HT, the sign of the photovoltage reverses and its size increases to $-160 \mathrm{mV}$ (Figure 4D). As we will show below, these changes are due to modification of the built-in voltage at the interlayer-P3HT interface. Interestingly, without thermal annealing of the $\mathrm{TiO}_{x}$

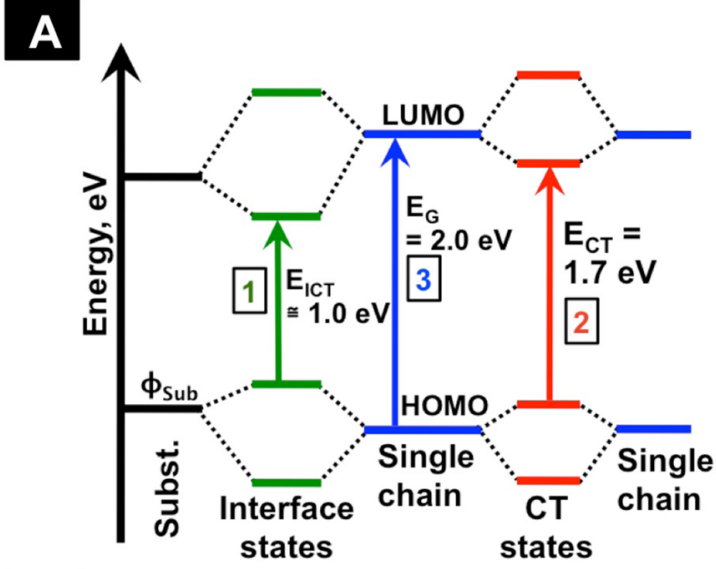

B


Polaron Pair

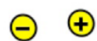

Free Charges

Charge Transfer

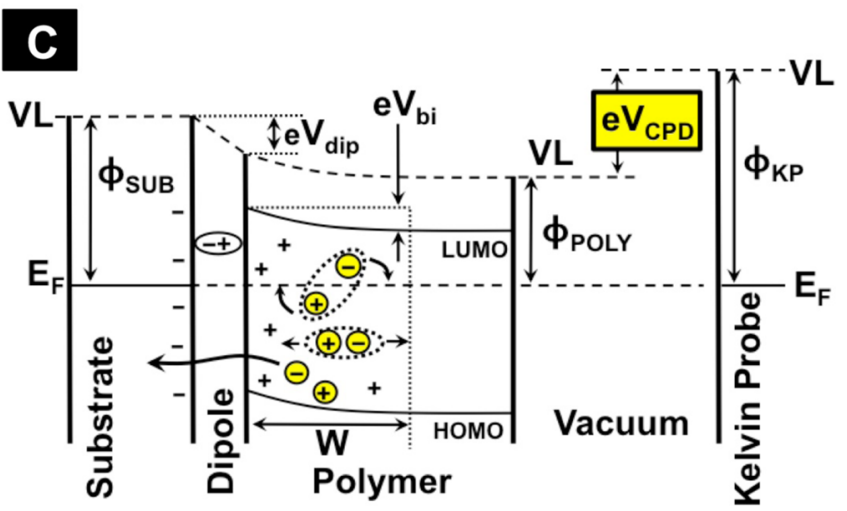

Figure 3. (A) Energy diagram for P3HT film and electronic transitions under illumination. (B) Morphology of P3HT film (see also Chen et al. ${ }^{58}$ and Roehling et al. ${ }^{59}$ ) including locations of excitons and CT states, F1 (interfacial charge transfer, ICT state), F2 (CT or autoionized state), F3 (band gap excited state). (C) Energy diagram for the P3HT film on ITO substrate (adapted from Ishii). ${ }^{60}$ For a low work function substrate like $\mathrm{TiO}_{2}$, the bands bend the opposite way. Photochemically generated charge carriers and polaron pairs move under the influence of electric fields created by the interfacial dipole and by Fermi level equilibration of substrate and P3HT. In addition, injection of charge carriers into the substrate is possible. These lightinduced processes alter the local vacuum level (VL) at the P3HT surface and cause the observed photovoltage, $\triangle$ CPD. $W$ : space charge layer width; $\phi_{i}$ : work functions of polymer, substrate, and Kelvin probe; $V_{\mathrm{bi}}$ : built-in voltage; $V_{\text {Dip }}$ : potential drop from interfacial dipole; $E_{\mathrm{F}}$ : Fermi level.

interlayer, the photovoltage does not change in comparison to ITO, suggesting that a heat-induced chemical change is taking place at the interface. Similarly, heating of the PEDOT:PSS- 

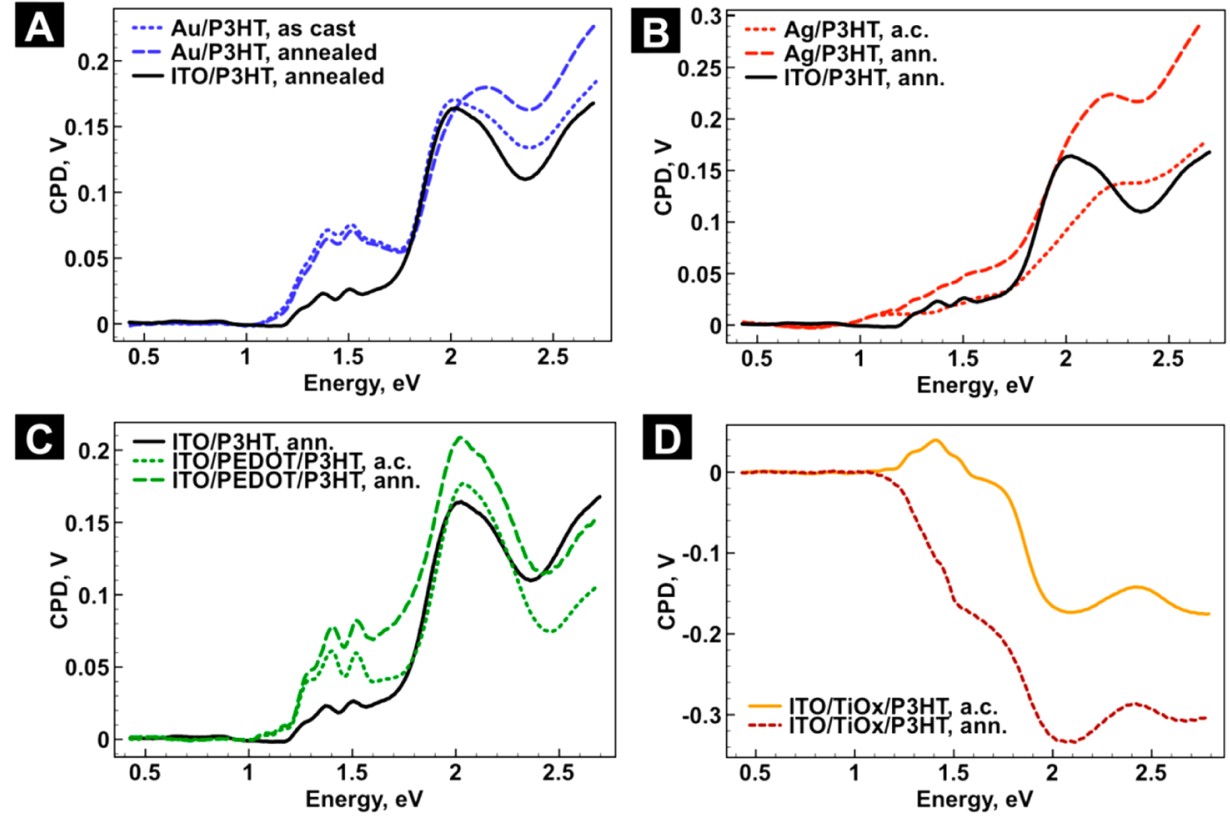

Figure 4. (A) SPV spectra of P3HT, as cast and after annealing, on $\mathrm{Au},(\mathrm{B})$ on $\mathrm{Ag},(\mathrm{C})$ on PEDOT:PSS, and (D) on sol-gel TiO $x_{x}$. All P3HT films had the same thickness. The annealed ITO sample spectrum is shown for comparison.

P3HT system results in an $75 \%$ voltage increase of F1 (Figure 4C). Previous work in our laboratory showed that thermal annealing promotes mixing between P3HT and PSS and formation of a doped interlayer. ${ }^{54} \mathrm{~A}$ final observation is that feature 1 is larger in rr-P3HT $(30 \mathrm{mV})$ than in rra-P3HT $(18$ $\mathrm{mV}$ ) (Figure 5A). This could be a result of the greater density of states of rr-P3HT near the Fermi level and consequentially a greater number of interfacial states.

Feature 2 is assigned to direct excitation of the autoionized charge transfer (CT) state in $\mathrm{P} 3 \mathrm{HT}$, based on its spectral overlap with the $\mathrm{P} 3 \mathrm{HT}$ film photoluminescence spectrum (Figure 2B). As expected, the photovoltage strongly depends on the thermal annealing state and the polymer isomer, rr- or rra-P3HT (Figure 5A). A change from rr- to rra-P3HT causes feature 2 to shift from 1.7 to $1.9 \mathrm{eV}$ and the photovoltage to drop from 110 to $30 \mathrm{mV}$ for the as-cast films and from 144 to 8 $\mathrm{mV}$ for the thermally annealed films. This is due to the lower concentration and larger HOMO-LUMO separation of CT states in rra-P3HT. The formation of the CT state is inhibited because the loose packing of the disordered (rra) polythiophene weakens the electronic interactions between chains. ${ }^{49,53}$ On the contrary, rr-P3HT has strong interchain $\pi-\pi$ interactions that produce CT states about $0.8 \mathrm{eV}$ below the polymer band gap. ${ }^{49,55}$ Thermal annealing of rr-P3HT films increases the fraction of crystalline $\mathrm{P} 3 \mathrm{HT}$ regions in the film ${ }^{56,57}$ and therefore raises the photovoltage (Figure 5A). In contrast, for rra-P3HT, thermal treatment leads to a reduction of the photovoltage because the annealed film is more homogeneous and so cannot support CT states at domain interfaces.

Feature 3 is assigned to direct band gap (EG) excitation of the P3HT. This is confirmed by the shift of the feature from 2.0 to $2.2 \mathrm{eV}$ in going from rr- to rra-P3HT (Figure 5A). The larger band gap of the latter can be seen in the absorption spectrum in the figure inset. It is a consequence of the greater isolation of electronic states in rra-P3HT, resulting from the loose packing of the polymer chains. This also causes a lower mobility of
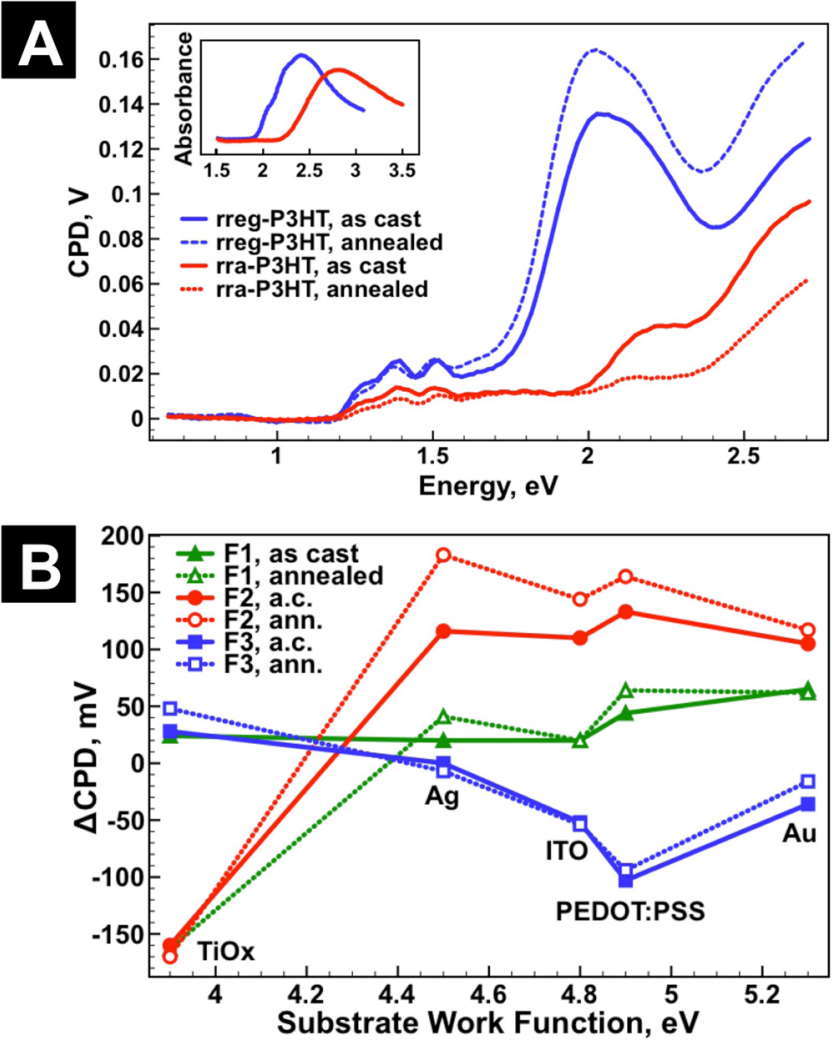

Figure 5. (A) SPV spectra of rr- and rra-P3HT films (ca. $120 \mathrm{~nm}$ thick) on ITO. Solid: as cast; dashed: after annealing. Inset: UV-vis absorption spectra of as cast rr- and rra-P3HT films. (B) SPV signal trends for as cast (solid) and for annealed rr-P3HT films (dotted) versus substrate work function. All data are from Table 1.

charge carriers in rra-P3HT and is the reason for the smaller size of F3 in the regiorandom film.

Wide-angle X-ray scattering, high-resolution X-ray reflectivity data, and TEM suggest that P3HT-PCBM bulk heterojunction 
films contain a mixture of amorphous and crystalline regions. ${ }^{58,61}$ On the basis of the signal interpretation above, photovoltage features $1-3$ can then be assigned to discrete regions in the P3HT film, as shown in Figure 3B. Surface photovoltage feature 1 originates from states at the polymersubstrate interface. The number of these states is relatively small due to the spatial restriction to the interface. Photovoltage feature 2 involves states between the amorphous and crystalline regions of the P3HT. Here the interchain interactions are stronger due to efficient packing of the chains. We hypothesize that the hole is stabilized in the crystalline regions while the electron resides either in the amorphous domain or at the domain interface. Lastly, surface photovoltage feature 3 originates from both amorphous and crystalline regions in the film. The formed carriers are free to move through the film and to pass through the substrate-polymer interface, as shown in Figure $3 \mathrm{~B}$, thus giving rise to the observed photovoltage.

Next we examine the physical reason for the observed lightinduced charge separation in the polymer film. According to the depleted semiconductor model, ${ }^{62}$ charge carriers in the excited P3HT film move under a built-in electric field that results from the electrochemical equilibrium between P3HT and the substrate and from dipoles at the substrate-P3HT interface (Figure 3C). ${ }^{35,60,63}$ An intrafilm surface photovoltage can be generated either by polarization of Coulombically bound electron-hole pairs (polarons) or by movement of free charge carriers through the P3HT film. Carrier movement stops when the electric field that acts on the film has been neutralized, and the potential variation across the P3HT film becomes flat. Additionally, an interfacial photovoltage can occur by selective transfer of free charge carriers across the film-substrate interface. Such charge transfer is driven by the concentration of free carriers and their chemical potential, and it depends on the overlap of wave functions between substrate and polymer at the interface. ${ }^{64}$ According to this interpretation, SPV features 1 and 2 are assigned to intrafilm polarization processes and SPV feature 3 to a charge transfer process within the film and through the polymer-substrate interface. Support for this assignment comes from a deeper analysis on the dependence of the SPV features on substrate, film thickness, and light intensity.

Figure 5B plots all photovoltage signals as a function of the substrate work function before and after annealing. Several observations can be made: Except for $\mathrm{TiO}_{x}, \mathrm{~F} 1$ and F2 are positive (negative side of polaron points to film surface), while F3 is negative (electrons move to substrate). Annealing does increase F2 but has only minor effects on F1 and F3. Only F1 and F3 display a systematic increase with the substrate work function, whereas $\mathrm{F} 2$ shows more erratic behavior. The $\mathrm{TiO}_{x}-$ coated substrate causes inversion of the photovoltage sign for all SPV features.

This data can be explained with the model in Figure 3C. Charge carriers created under subgap illumination (1 and 2) are bound together by Coulomb forces. They cannot move by drift under the built-in electric field but they can orient/distort under it, producing a photovoltage. The built-in field at the substrate-polymer interface is a function of the Fermi level difference between the substrate and the P3HT film, which explains the variation of feature 1 with the substrate work function. Polaron pairs generated under CT excitation (feature 2) also orient in the direction of the field generated at the substrate-polymer interface. The size of feature 2 , however, is not only a function of the field but also of the polaron concentration in the film, as determined by the generation and recombination rates-hence the strong dependence on thermal annealing, which promotes these carriers through the creation of more interfaces between crystalline and amorphous domains. Charge carriers created under band-gap excitation (feature 3) are free and can diffuse through the P3HT film and inject into the substrate, as long as their electrochemical potential exceeds that of the substrate. ${ }^{35}$ This explains the increase of the size of F3 with increasing substrate work function. However, the true size of F3 is masked by the cocreation of bound CT states (F1 and F2) that oppose the field created by free charges. A notable exception for the work function dependence occurs for the gold substrate. Tentatively, we attribute this to optical excitation of the gold plasmon band $(>2.4 \mathrm{eV})$. Plasmon resonance-induced charge transfer processes have been previously hypothesized for silver nanoprisms. ${ }^{65}$ Among all substrates, the $\mathrm{TiO}_{x}$ interlayer has the lowest work function. Using the model in Figure 3C, this inverts the electrical field at the $\mathrm{TiO}_{x}-\mathrm{P} 3 \mathrm{HT}$ interface and hence all photovoltage signals. Based on the inversion point, the effective work function of $\mathrm{P} 3 \mathrm{HT}$ is between 4.5 and $4.2 \mathrm{eV}$. We note that the work function of P3HT has been measured at 4.2-4.5 eV using both ultraviolet photoelectron spectroscopy and Kelvin force probe measurements. ${ }^{66-68}$ It is not at $5.2 \mathrm{eV}$ as commonly stated, but rather this is the HOMO level of P3HT. 69

Table 1. Experimental $\triangle \mathrm{CPD}$ Values for rr-P3HT Films on Various Substrates

\begin{tabular}{|c|c|c|c|c|}
\hline substrate & $\begin{array}{c}\text { work function } \\
{[\mathrm{eV}]}\end{array}$ & $\begin{array}{c}\mathrm{F} 1 \\
{[\mathrm{mV}]}\end{array}$ & $\begin{array}{c}\mathrm{F} 2 \\
{[\mathrm{mV}]}\end{array}$ & $\begin{array}{c}\mathrm{F} 3 \\
{[\mathrm{mV}]}\end{array}$ \\
\hline $\mathrm{Au}$, as cast & $5.3^{a}$ & 65 & 105 & -36 \\
\hline PEDOT:PSS, as cast & $5.0^{b}$ & 44 & 133 & -103 \\
\hline ITO, as cast & $4.8^{a}$ & 20 & 110 & -52 \\
\hline $\mathrm{Ag}$, as cast & $4.5^{a}$ & 20 & 116 & 0 \\
\hline $\mathrm{TiO}_{x}$, as cast & $3.9^{c}$ & 24 & -160 & 28 \\
\hline $\mathrm{Au}$, annealed & $5.3^{a}$ & 62 & 117 & -16 \\
\hline PEDOT:PSS, annealed & $5.0^{b}$ & 64 & 164 & -94 \\
\hline ITO, annealed & $4.8^{a}$ & 20 & 144 & -54 \\
\hline Ag, annealed & $4.5^{a}$ & 41 & 183 & -7 \\
\hline $\mathrm{TiO}_{x}$, annealed & $3.9^{c}$ & -163 & -170 & 48 \\
\hline
\end{tabular}

${ }^{a}$ From CRC Handbook of Chemistry and Physics. ${ }^{70}{ }^{b}$ From Xu et al. ${ }^{71}$ ${ }^{c}$ Measured with Kelvin probe.

The dependence of the SPV signal and optical absorption on film thickness is explored in Figure 6 for as cast and annealed films on ITO. Overall, photovoltage features only weakly depend on film thickness, especially when the films are above $120 \mathrm{~nm}$. For as-cast films (feature 6A), F1 and F2 mildly decrease and F3 mildly increases. Annealing (feature 6B) reverses the trends for $\mathrm{F} 1$ and $\mathrm{F} 2$, which now slowly decrease with thickness, but not for F3 (feature 6C).

The data conform well to the model in Figure 3C. Photovoltage features 1 and 2 are controlled by the built-infield at the substrate-polymer interface, which changes little with film thickness. In contrast, F3 is limited by the concentration of free charge carriers in the film, which depends not on film thickness, but on film quality, as the latter controls the exciton lifetime via the concentration of recombination sites. This dependence also explains the near doubling of the F3 signal upon annealing. The observed changes in F1 and F2 
A
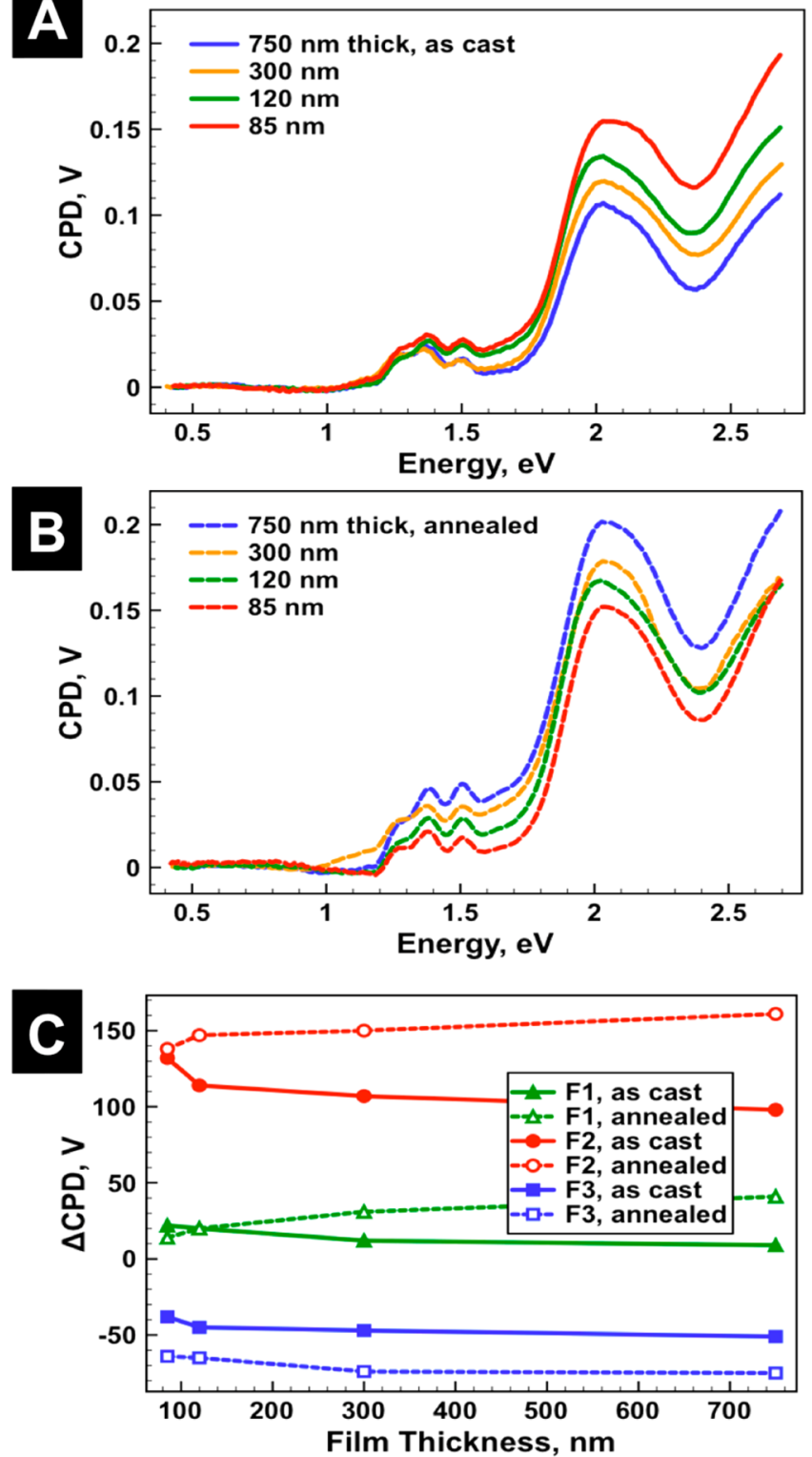

Figure 6. SPV spectra on variable thickness as-cast (A) and thermally annealed (B) rr-P3HT films on ITO. (C) $\triangle$ CPD values for features 1, 2 , and 3 (measured from the beginning to the end of each feature).

upon annealing are due to modification of the polymer substrate interface, as discussed above.

Lastly, to determine the effect of illumination on charge separation, variable intensity SPV measurements were conducted on as-cast and annealed rr-P3HT films on ITO. Figure 7A shows SPV spectra for several illumination intensities, while the graph in Figure 7B shows the dependence of individual photovoltage features on the incident photon flux. On a per photon basis, the photovoltage signals decrease in the order F2 $>$ F3 > F1. Feature 1 linearly increases with photon flux, whereas F2 and F3 show a near-logarithmic dependence on the flux. Annealing increases F3, especially at high photon densities, and F2 at intermediate photon densities. Annealing does not increase F1. These results support the basic assumptions of the model in Figure 3. As an interfacial process, F1 is limited by the finite density of states at the polymer-substrate interface. Thus, it is less dependent on the number of incident photons. Feature
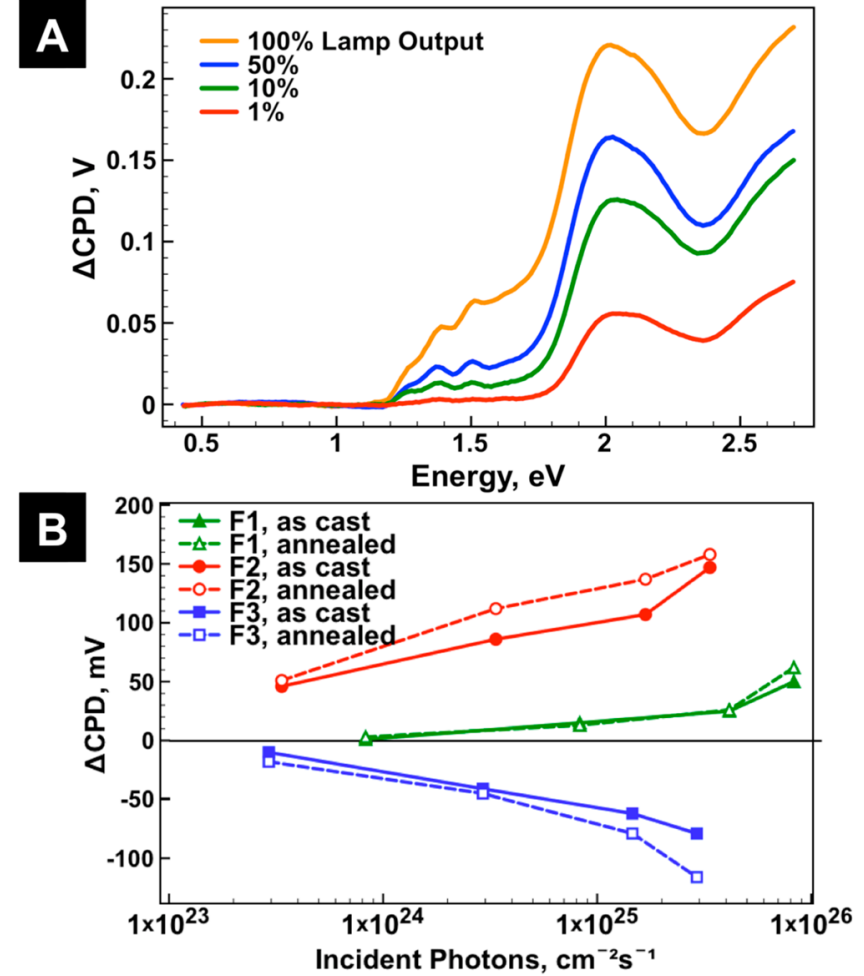

Figure 7. (A) SPV spectra for $120 \mathrm{~nm}$ as-cast rr-P3HT on ITO. (B) Relative size of photovoltage signals for F1-F3 before (solid lines) and after annealing (dashed lines) versus incident light flux. $\triangle \mathrm{CPD}$ values were extracted from the full spectra at $1.45,1.90$, and $2.20 \mathrm{eV}$.

2 depends on the number of states in and around the P3HT crystal domains and so increases upon annealing. Because the absorption cross section of these sub-band-gap states is very low, a strong increase with photon flux is observed. On the contrary, the size of F3 is mostly determined by the generation-recombination equilibrium of the free charge carriers. For this situation, the Shockley diode equation ${ }^{7 / 2}$ predicts the observed logarithmic dependence on the photon flux. Thermal annealing raises the carrier concentrations by reducing defect concentrations in the polymer film.

\section{CONCLUSION}

In summary, we present surface photovoltage spectra of P3HT films, as a function of polymer type (rr- or rra-), film thickness, substrate type, and illumination intensity. Three different photovoltage processes could be resolved as a function of the excitation energy and are assigned to distinct polymer states in the film. This includes excitation of interfacial states at the substrate P3HT interface (F1), excitation of CT states between P3HT chains (F2), and excitation of the band gap transition of individual P3HT chains (F3). Only F3 is due to motion of free charge carriers, whereas F1 and F2 are due to polarization of Coulombically bound polarons from the electric field at the substrate-polymer interface. The dependence of these features on film thickness, substrate work function, and polymer type can be explained with the depleted semiconductor model in Figure 3C.

The results confirm that the CT state can be effectively populated with light of sub-band-gap energy. They also highlight the importance of interfacial and CT states in attenuating the electric field across the organic film. And they emphasize the ability of $\mathrm{TiO}_{x}$ and PEDOT:PSS layers to 
modify the electric field at the substrate polymer interface and thus to control the orientation of the photodipoles. Lastly, this work shows that SPS is a useful technique for the characterization of charge separation in organic films. Results of a SPS study on P3HT-PCBM bulk heterojunctions will be reported in a separate paper.

\section{EXPERIMENTAL SECTION}

UV-vis absorption measurements were performed using a Thermo Scientific Evolution $220 \mathrm{UV}$-vis spectrophotometer equipped with an integrating sphere. Film thicknesses were determined with a Dektak surface profiler calibrated to a $\mathrm{Si}-$ $\mathrm{SiO}_{2}$ ellipsometry standard. Monochromatic light intensity measurements were performed using an International Light Technologies IL400BL photometer with an IR-visible detector. Work function measurements were made under ambient conditions using a Kelvin probe from KP Technologies, Inc. Surface photovoltage spectra (SPS) were recorded on a home-built instrument that utilizes a Kelvin probe and controller (Besocke Delta Phi GmbH), a custombuilt high vacuum measurement chamber, a voltmeter (Keithley 2700), a monochromator (Oriel Cornerstone 130), a Pfeiffer HiCube 80 Eco turbo pump, a $100 \mathrm{~W}$ Xe arc lamp as the light source, and a neutral density optical filter. For the measurements, samples were mounted inside the vacuum chamber, and the Kelvin probe (Au grid, $3 \mathrm{~mm}$ diameter, 60\% transparent) was placed $1.0 \mathrm{~mm}$ above the sample. The chamber was evacuated to $10^{-7}$ bar, and steady-state photovoltage spectra were obtained by scanning from low- to high-energy radiation in $100 \mathrm{~cm}^{-1}$ steps with a period interval of $5 \mathrm{~s}$. Light spectra were corrected for background effects (drift, desorption of gas and solvent molecules) by subtracting a background scan in the dark. The sign convention is chosen so that a positive $\triangle \mathrm{CPD}$ value will correspond to dipole with a negative pole at the surface and the positive side toward the back electrode. Based on repeat measurements, the approximate voltage error is $10 \%$ and the approximate photon energy error is $0.05 \mathrm{eV}$. All film samples were prepared using commercial indium-tin oxide (ITO) coated glass (ca. $140 \mathrm{~nm}$ thick and $15 \Omega$ sheet resistance), unless noted otherwise. The ITO was cleaned by scrubbing with acetone and exposed to UV-ozone for $30 \mathrm{~min}$ before use. For the substrate variation tests commercial goldcoated glass (Thermo Scientific, ca. $80 \mathrm{~nm}$ ) was cleaned with the same method above before use. Silver-coated glass substrates $(100 \mathrm{~nm})$ were prepared in-house by vapor deposition. Poly(3-hexylthiophene) (P3HT) was generously donated by Plextronics Inc., with $\mathrm{MW} \approx 70 \mathrm{kDa}$ and a reported regioregularity of $\geq 95 \%$; regiorandom $\mathrm{P} 3 \mathrm{HT}$ with $\mathrm{MW} \approx 40$ $\mathrm{kDa}$ (50\% regioregular) was purchased from Aldrich. Polymers were dissolved in chlorobenzene (Fisher Scientific, >99.9\%) at $60{ }^{\circ} \mathrm{C}$ overnight $(20 \mathrm{mg} / \mathrm{mL})$, and the solutions were spincoated $(800 \mathrm{rpm})$ under a nitrogen atmosphere to give $\sim 120$ $\mathrm{nm}$ thick films. The thickest P3HT film (ca. $750 \pm 100 \mathrm{~nm}$ ) was drop-cast from solution. Each of the films was tested before and after annealing. Annealing was performed under a nitrogen atmosphere at $120{ }^{\circ} \mathrm{C}$ for $10 \mathrm{~min}$. $\mathrm{TiO}_{x}$ sol-gel was synthesized from titanium isopropoxide (TIP, 97\%, SigmaAldrich) according to the published procedure ${ }^{73}$ and diluted in methanol for spin-coating to desired thicknesses. In certain cases, samples were spin-coated with $40 \mathrm{~nm}$ of PEDOT:PSS (Clevios P AI 4083).

\section{AUTHOR INFORMATION}

\section{Corresponding Author}

*E-mail: fosterloh@ucdavis.edu; Ph (530) 754-6242 (F.E.O.).

\section{Notes}

The authors declare no competing financial interest.

\section{ACKNOWLEDGMENTS}

This work was supported by a grant of the National Science Foundation (NSF, CBET 933435) and by a Scialog grant from Research Corporation for Science Advancement.

\section{REFERENCES}

(1) Clarke, T. M.; Durrant, J. R. Charge Photogeneration in Organic Solar Cells. Chem. Rev. 2010, 110, 6736-6767.

(2) Deibel, C.; Dyakonov, V. Polymer-Fullerene Bulk Heterojunction Solar Cells. Rep. Prog. Phys. 2010, 73, 096401.

(3) Schlenker, C. W.; Thompson, M. E. The Molecular Nature of Photovoltage Losses in Organic Solar Cells. Chem. Commun. 2011, 47, 3702-3716.

(4) Moule, A. J. Power from Plastic. Curr. Opin. Solid State Mater. Sci. 2010, 14, 123-130.

(5) Mayer, A. C.; Scully, S. R.; Hardin, B. E.; Rowell, M. W.; McGehee, M. D. Polymer-Based Solar Cells. Mater. Today 2007, 10, $28-33$.

(6) Yu, G.; Gao, J.; Hummelen, J. C.; Wudl, F.; Heeger, A. J. Polymer Photovoltaic Cells: Enhanced Efficiencies Via a Network of Internal Donor-Acceptor Heterojunctions. Science 1995, 270, 1789-1791.

(7) Schlenker, C. W.; Thompson, M. E. The Molecular Nature of Photovoltage Losses in Organic Solar Cells. Chem. Commun. 2011, 47, 3702-3716.

(8) Hiramoto, M.; Fujiwara, H.; Yokoyama, M. P-I-N Like Behavior in Threelayered Organic Solar Cells Having a Codeposited Interlayer of Pigments. J. Appl. Phys. 1992, 72, 3781-3787.

(9) Halls, J. J. M.; Walsh, C. A.; Greenham, N. C.; Marseglia, E. A.; Friend, R. H.; Moratti, S. C.; Holmes, A. B. Efficient Photodiodes from Interpenetrating Polymer Networks. Nature 1995, 376, 498-500.

(10) Shaheen, S. E.; Brabec, C. J.; Sariciftci, N. S.; Padinger, F.; Fromherz, T.; Hummelen, J. C. 2.5\% Efficient Organic Plastic Solar Cells. Appl. Phys. Lett. 2001, 78, 841-843.

(11) Street, R.; Davies, D.; Khlyabich, P.; Burkhart, B.; Thompson, B. Origin of the Tunable Open-Circuit Voltage in Ternary Blend Bulk Heterojunction Organic Solar Cells. J. Am. Chem. Soc. 2013, 135, 986989.

(12) Vandewal, K.; Tvingstedt, K.; Gadisa, A.; Inganas, O.; Manca, J. V. On the Origin of the Open-Circuit Voltage of Polymer-Fullerene Solar Cells. Nat. Mater. 2009, 8, 904-909.

(13) Vandewal, K.; Gadisa, A.; Oosterbaan, W. D.; Bertho, S.; Banishoeib, F.; Van Severen, I.; Lutsen, L.; Cleij, T. J.; Vanderzande, D.; Manca, J. V. The Relation between Open-Circuit Voltage and the Onset of Photocurrent Generation by Charge-Transfer Absorption in Polymer: Fullerene Bulk Heterojunction Solar Cells. Adv. Funct. Mater. 2008, 18, 2064-2070.

(14) Brabec, C. J.; Cravino, A.; Meissner, D.; Sariciftci, N. S.; Fromherz, T.; Rispens, M. T.; Sanchez, L.; Hummelen, J. C. Origin of the Open Circuit Voltage of Plastic Solar Cells. Adv. Funct. Mater. 2001, 11, 374-380.

(15) Street, R. A.; Davies, D.; Khlyabich, P. P.; Burkhart, B.; Thompson, B. C. Origin of the Tunable Open-Circuit Voltage in Ternary Blend Bulk Heterojunction Organic Solar Cells. J. Am. Chem. Soc. 2013, 135, 986-989.

(16) Dimitrov, S. D.; Bakulin, A. A.; Nielsen, C. B.; Schroeder, B. C.; Du, J. P.; Bronstein, H.; McCulloch, I.; Friend, R. H.; Durrant, J. R. On the Energetic Dependence of Charge Separation in Low-Band-Gap Polymer/Fullerene Blends. J. Am. Chem. Soc. 2012, 134, 1818918192. 
(17) Cowan, S. R.; Roy, A.; Heeger, A. J. Recombination in PolymerFullerene Bulk Heterojunction Solar Cells. Phys. Rev. B 2010, 82, 245207.

(18) Hallermann, M.; Da Como, E. Ct States in Meh-Ppv/Pcbm Blends: Below-Gap Wealy Bound Excitons for Polymer Photovoltaics. Appl. Phys. Lett. 2008, 93, 053307.

(19) Janssen, R. A. J.; Veldman, D. The Energy of Charge-Transfer States in Electron Donor-Acceptor Blends: Insight into the Energy Losses in Organic Solar Cells. Adv. Funct. Mater. 2009, 19, 19391948.

(20) Hamilton, R.; Shuttle, C. G.; O’Regan, B.; Hammant, T. C.; Nelson, J.; Durrant, J. R. Recombination in Annealed and Nonannealed Polythiophene/Fullerene Solar Cells: Transient Photovoltage Studies Versus Numerical Modeling. J. Phys. Chem. Lett. 2010, 1, 1432-1436.

(21) Liang, Z.; Gregg, B. A. Compensating Poly(3-Hexylthiophene) Reveals Its Doping Density and Its Strong Exciton Quenching by Free Carriers. Adv. Mater. 2012, 24, 3258-3262.

(22) Belijonne, D.; Sirringhaus, H.; Brown, P. J.; Friend, R. H.; Bredas, J. L. Optical Signature of Delocalized Polarons in Conjugated Polymers. Adv. Funct. Mater. 2001, 11, 229-234.

(23) Tamura, H.; Tsukada, M. Role of Intermolecular Charge Delocalization on Electron Transport in Fullerene Aggregates. Phys. Rev. B 2012, 85, 054301.

(24) Bisquert, J.; Garcia-Belmonte, G.; Munar, A.; Sessolo, M.; Soriano, A.; Bolink, H. J. Band Unpinning and Photovoltaic Model for P3ht:Pcbm Organic Bulk Heterojunctions under Illumination. Chem. Phys. Lett. 2008, 465, 57-62.

(25) Hwang, J.; Wan, A.; Kahn, A. Energetics of Metal-Organic Interfaces: New Experiments and Assessment of the Field. Mater. Sci. Eng., $R$ 2009, 64, 1-31.

(26) Vandewal, K.; Gadisa, A.; Oosterbaan, W. D.; Bertho, S.; Banishoeib, F.; Van Severen, I.; Lutsen, L.; Cleij, T. J.; Van der Zande, D.; Manca, J. V. The Relation between Open-Circuit Voltage and the Onset of Photocurrent Generation by Charge-Transfer Absorption in Polymer: Fullerene Bulk Heterojunction Solar Cells. Adv. Funct. Mater. 2008, 18, 2064-2070.

(27) Nelson, J.; Durrant, J. R.; Poplavskyy, D. Ambipolar Charge Transport in Films of Methanofullerene and Poly(Phenylenevinylene)/Methanofullerene Blends. Adv. Funct. Mater. 2005, 15, 1171-1182.

(28) Tvingstedt, K.; Inganas, O.; Manca, J. V. Electroluminescence from Charge Transfer States in Polymer Solar Cells. J. Am. Chem. Soc. 2009, 131, 11819-11824.

(29) Sirringhaus, H.; Brown, P. J.; Friend, R. H.; Nielsen, M. M.; Bechgaard, K.; Langeveld-Voss, B. M. W.; Spiering, A. J. H.; Janssen, R. A. J.; Meijer, E. W.; Herwig, P.; et al. Two-Dimensional Charge Transport in Self-Organized, High-Mobility Conjugated Polymers. Nature 1999, 401, 685-688.

(30) Kronik, L.; Shapira, Y. Surface Photovoltage Phenomena: Theory, Experiment, and Applications. Surf. Sci. Rep. 1999, 37, 1-206.

(31) Dittrich, T.; Fiechter, S.; Thomas, A. Surface Photovoltage Spectroscopy of Carbon Nitride Powder. Appl. Phys. Lett. 2011, 99, 084105.

(32) Beranek, R.; Neumann, B.; Sakthivel, S.; Janczarek, M.; Dittrich, T.; Tributsch, H.; Kisch, H. Exploring the Electronic Structure of Nitrogen-Modified $\mathrm{TiO}_{2}$ Photocatalysts through Photocurrent and Surface Photovoltage Studies. Chem. Phys. 2007, 339, 11-19.

(33) Gross, D.; Mora-Sero, I.; Dittrich, T.; Belaidi, A.; Mauser, C.; Houtepen, A. J.; Da Como, E.; Rogach, A. L.; Feldmann, J. Charge Separation in Type II Tunneling Multi Layered Structures of CdTe and CdSe Nanocrystals Directly Proven by Surface Photovoltage Spectroscopy. J. Am. Chem. Soc. 2010, 132, 5981.

(34) Zabel, P.; Dittrich, T.; Funes, M.; Durantini, E. N.; Otero, L. Charge Separation at Pd-Porphyrin/ $/ \mathrm{TiO}_{2}$ Interfaces. J. Phys. Chem. C 2009, 113, 21090-21096.

(35) Kronik, L.; Shapira, Y. Surface Photovoltage Spectroscopy of Semiconductor Structures: At the Crossroads of Physics, Chemistry and Electrical Engineering. Surf. Interface Anal. 2001, 31, 954-965.
(36) Donchev, V.; Kirilov, K.; Ivanov, T.; Germanova, K. Surface Photovoltage Phase Spectroscopy - a Handy Tool for Characterisation of Bulk Semiconductors and Nanostructures. Mater. Sci. Eng., B 2006, 129, 186-192.

(37) Dahlberg, S. C.; Musser, M. E. Surface Photo-Voltage of an Increasing Series of Polyarenes - Anthracene, Tetracene, and Pentacene. J. Chem. Phys. 1979, 71, 2806-2810.

(38) Musser, M. E.; Dahlberg, S. C. The Surface Photo-Voltage of Polymethine Semiconducting-Films. J. Chem. Phys. 1980, 72, 40844088.

(39) Mishori, B.; Shapira, Y.; BeluMarian, A.; Manciu, M.; Devenyi, A. Studies of C-60 Thin Films Using Surface Photovoltage Spectroscopy. Chem. Phys. Lett. 1997, 264, 163-167.

(40) Luria, J. L.; Hoepker, N.; Bruce, R.; Jacobs, A. R.; Groves, C.; Marohn, J. A. Spectroscopic Imaging of Photopotentials and Photoinduced Potential Fluctuations in a Bulk Heterojunction Solar Cell Film. ACS Nano 2012, 6, 9392-9401.

(41) Maturova, K.; Kemerink, M.; Wienk, M. M.; Charrier, D. S. H.; Janssen, R. A. J. Scanning Kelvin Probe Microscopy on Bulk Heterojunction Polymer Blends. Adv. Funct. Mater. 2009, 19, 13791386.

(42) Palermo, V.; Ridolfi, G.; Talarico, A. M.; Favaretto, L.; Barbarella, G.; Camaioni, N.; Samorì, P. A Kelvin Probe Force Microscopy Study of the Photogeneration of Surface Charges in AllThiophene Photovoltaic Blends. Adv. Funct. Mater. 2007, 17, 472478 .

(43) Liscio, A.; De Luca, G.; Nolde, F.; Palermo, V.; Muellen, K.; Samori, P. Photovoltaic Charge Generation Visualized at the Nanoscale: A Proof of Principle. J. Am. Chem. Soc. 2007, 130, 780781.

(44) Coffey, D. C.; Ginger, D. S. Time-Resolved Electrostatic Force Microscopy of Polymer Solar Cells. Nat. Mater. 2006, 5, 735-740.

(45) Padinger, F.; Rittberger, R. S.; Sariciftci, N. S. Effects of Postproduction Treatment on Plastic Solar Cells. Adv. Funct. Mater. 2003, 13, 85-88.

(46) Reyes-Reyes, M.; Kim, K.; Carroll, D. L. High-Efficiency Photovoltaic Devices Based on Annealed Poly(3-Hexylthiophene) and 1-(3-Methoxycarbonyl)-Propyl-1-Phenyl-(6,6)C-61 Blends. Appl. Phys. Lett. 2005, 87, 083506.

(47) Assadi, A.; Svensson, C.; Willander, M.; Inganas, O. Field-Effect Mobility of Poly(3-Hexylthiophene). Appl. Phys. Lett. 1988, 53, 195197.

(48) Zen, A.; Pflaum, J.; Hirschmann, S.; Zhuang, W.; Jaiser, F.; Asawapirom, U.; Rabe, J. P.; Scherf, U.; Neher, D. Effect of Molecular Weight and Annealing of Poly(3-Hexylthiophene)s on the Performance of Organic Field-Effect Transistors. Adv. Funct. Mater. 2004, 14, $757-764$.

(49) Guo, J. M.; Ohkita, H.; Benten, H.; Ito, S. Near-IR Femtosecond Transient Absorption Spectroscopy of Ultrafast Polaron and Triplet Exciton Formation in Polythiophene Films with Different Regioregularities. J. Am. Chem. Soc. 2009, 131, 16869-16880.

(50) Hoppe, H.; Sariciftci, N. S. Morphology of Polymer/Fullerene Bulk Heterojunction Solar Cells. J. Mater. Chem. 2006, 16, 45-61.

(51) Kozub, D. R.; Vakhshouri, K.; Orme, L. M.; Wang, C.; Hexemer, A.; Gomez, E. D. Polymer Crystallization of Partially Miscible Polythiophene/Fullerene Mixtures Controls Morphology. Macromolecules 2011, 44, 5722-5726.

(52) Cook, S.; Furube, A.; Katoh, R. Analysis of the Excited States of Regioregular Polythiophene P3ht. Energy Environ. Sci. 2008, 1, 294299.

(53) Korovyanko, O. J.; Osterbacka, R.; Jiang, X. M.; Vardeny, Z. V.; Janssen, R. A. J. Photoexcitation Dynamics in Regioregular and Regiorandom Polythiophene Films. Phys. Rev. B 2001, 64, 235122.

(54) Mauger, S. A.; Chang, L. L.; Rochester, C. W.; Moule, A. J. Directional Dependence of Electron Blocking in PEDOT:PSS. Org. Electron. 2012, 13, 2747-2756.

(55) Maillard, A.; Rochefort, A. Structural and Electronic Properties of Poly(3-Hexylthiophene) Pi-Stacked Crystals. Phys. Rev. B 2009, 79, 115207. 
(56) Ma, W. L.; Yang, C. Y.; Gong, X.; Lee, K.; Heeger, A. J. Thermally Stable, Efficient Polymer Solar Cells with Nanoscale Control of the Interpenetrating Network Morphology. Adv. Funct. Mater. 2005, 15, 1617-1622.

(57) Gao, Y. Q.; Grey, J. K. Resonance Chemical Imaging of Polythiophene/Fullerene Photovoltaic Thin Films: Mapping Morphology-Dependent Aggregated and Unaggregated $\mathrm{C}=\mathrm{C}$ Species. J. Am. Chem. Soc. 2009, 131, 9654-9662.

(58) Chen, W.; Xu, T.; He, F.; Wang, W.; Wang, C.; Strzalka, J.; Liu, Y.; Wen, J. G.; Miller, D. J.; Chen, J. H.; et al. Hierarchical Nanomorphologies Promote Exciton Dissociation in Polymer/Fullerene Bulk Heterojunction Solar Cells. Nano Lett. 2011, 11, 37073713.

(59) Roehling, J. D.; Batenburg, K. J.; Swain, F. B.; Moulé, A. J.; Arslan, I. Three-Dimensional Concentration Mapping of Organic Blends. Adv. Funct. Mater. 2013, 23, 2115-2122.

(60) Ishii, H.; Sugiyama, K.; Ito, E.; Seki, K. Energy Level Alignment and Interfacial Electronic Structures at Organic Metal and Organic Organic Interfaces. Adv. Mater. 1999, 11, 605.

(61) Roehling, J. D.; Batenburg, K. J.; Swain, F. B.; Moulé, A. J.; Arslan, I. Three-Dimensional Concentration Mapping of Organic Blends. Adv. Funct. Mater. 2013, 23, 2115-2122.

(62) Würfel, P. Physics of Solar Cells from Principles to New Concepts; Wiley-VCH: Weinheim, 2005.

(63) Fefer, E.; Kronik, L.; Leibovitch, M.; Shapira, Y.; Riedl, W. InSitu Monitoring of Surface Chemistry and Charge Transfer at Semiconductor Surfaces. Appl. Surf. Sci. 1996, 104, 61-67.

(64) Tvrdy, K.; Frantsuzov, P. A.; Kamat, P. V. Photoinduced Electron Transfer from Semiconductor Quantum Dots to Metal Oxide Nanoparticles. Proc. Natl. Acad. Sci. U. S. A. 2011, 108, 29-34.

(65) Wu, X. M.; Redmond, P. L.; Liu, H. T.; Chen, Y. H.; Steigerwald, M.; Brus, L. Photovoltage Mechanism for Room Light Conversion of Citrate Stabilized Silver Nanocrystal Seeds to Large Nanoprisms. J. Am. Chem. Soc. 2008, 130, 9500-9506.

(66) Fahlman, M.; Crispin, A.; Crispin, X.; Henze, S. K. M.; Jong, M. P. d.; Osikowicz, W.; Tengstedt, C.; Salaneck, W. R. Electronic Structure of Hybrid Interfaces for Polymer-Based Electronics. J. Phys.: Condens. Matter 2007, 19, 183202.

(67) Tengstedt, C.; Osikowicz, W.; Salaneck, W. R.; Parker, I. D.; Hsu, C. H.; Fahlman, M. Fermi-Level Pinning at Conjugated Polymer Interfaces. Appl. Phys. Lett. 2006, 88, 053502.

(68) Braun, S.; Salaneck, W. R.; Fahlman, M. Energy-Level Alignment at Organic/Metal and Organic/Organic Interfaces. Adv. Mater. 2009, 21, 1450-1472.

(69) Berson, S.; de Bettignies, R.; Bailly, S.; Guillerez, S.; Jousselme, B. Elaboration of P3HT/CNT/PCBM Composites for Organic Photovoltaic Cells. Adv. Funct. Mater. 2007, 17, 3363-3370.

(70) Lide, D. R. In CRC Handbook of Chemistry and Physics, 88th (Internet Version 2008) ed.; CRC Press/Taylor and Francis: Boca Raton, FL, 2008.

(71) Xu, Y.; Schoonen, M. A. A. The Absolute Energy Positions of Conduction and Valence Bands of Selected Semiconducting Minerals. Am. Mineral. 2000, 85, 543-556.

(72) Shockley, W. The Theory of P-N Junctions in Semiconductors and P-N Junction Transistors. Bell Syst. Tech. J. 1949, 28, 435-489.

(73) Wang, D. H.; Im, S. H.; Lee, H. K.; Park, O. O.; Park, J. H. Enhanced High-Temperature Long-Term Stability of Polymer Solar Cells with a Thermally Stable $\mathrm{TiO}_{\mathrm{x}}$ Interlayer. J. Phys. Chem. C 2009, 113, 17268-17273. 